\title{
M-Channel Activation Contributes to the Anticonvulsant Action of the Ketone Body $\beta$-Hydroxybutyrate ${ }^{\text {S }}$
}

\author{
Rían W. Manville, Maria Papanikolaou, and Geoffrey W. Abbott \\ Bioelectricity Laboratory, Department of Physiology and Biophysics, School of Medicine, University of California, Irvine, \\ California
}

Received October 20, 2019; accepted November 20, 2019

\begin{abstract}
Ketogenic diets are effective therapies for refractory epilepsy, yet the underlying mechanisms are incompletely understood. The anticonvulsant efficacy of ketogenic diets correlates positively to the serum concentration of $\beta$-hydroxybutyrate $(\mathrm{BHB})$, the primary ketone body generated by ketosis. Voltage-gated potassium channels generated by KCNQ2-5 subunits, especially KCNQ2/3 heteromers, generate the M-current, a therapeutic target for synthetic anticonvulsants. Here, we report that $\mathrm{BHB}$ directly activates $\mathrm{KCNQ} 2 / 3$ channels $\left(\mathrm{EC}_{50}=0.7 \mu \mathrm{M}\right)$, via a highly conserved S5 tryptophan (W265) on KCNQ3. BHB was also acutely effective as an anticonvulsant in the pentylene tetrazole (PTZ) seizure assay in mice. Strikingly, coadministration of $\gamma$-amino- $\beta$-hydroxybutyric acid, a high-affinity KCNQ2/3 partial agonist that also acts via KCNQ3-W265, similarly reduced the
\end{abstract}

efficacy of BHB in KCNQ2/3 channel activation in vitro and in the PTZ seizure assay in vivo. Our results uncover a novel, unexpected molecular basis for anticonvulsant effects of the major ketone body induced by ketosis.

\section{SIGNIFICANCE STATEMENT}

Ketogenic diets are used to treat refractory epilepsy but the therapeutic mechanism is not fully understood. Here, we show that clinically relevant concentrations of $\beta$-hydroxybutyrate, the primary ketone body generated during ketogenesis, activates KCNQ2/3 potassium channels by binding to a specific site on KCNQ3, an effect known to reduce neuronal excitability. We provide evidence using a mouse chemoconvulsant model that KCNQ2/3 activation contributes to the antiepileptic action of $\beta$-hydroxybutyrate.

\section{Introduction}

Epilepsy remains a major, inadequately controlled neurologic disorder, affecting around 50 million individuals globally, $\sim 20 \%$ of whom are refractory to medications (Shorvon, 1996). A number of inherited and other disorders lie under the umbrella term epilepsy; however, a common thread is inadequate control of neuronal excitability, leading to seizures ranging from relatively benign and transient to life threatening and severe (Falco-Walter et al., 2018). Aside from medication, one intervention currently attracting renewed interest, especially for treatment of refractory pediatric epilepsy, is the ketogenic diet (Nordli, 2009). The ketogenic diet is a lowcarbohydrate, high-fat, adequate-protein diet regimen that deprives the body of dietary glucose, which in a normal diet is a particularly important fuel source for energy-hungry neuronal activity. Faced with a low-carbohydrate, high-fat diet, hepatocytes in the liver increasingly convert fats into fatty acids and ketone bodies, which enter the brain via the blood

This study was supported by the National Institutes of Health National Institute of General Medical Sciences [Grant GM115189] and National Institute of Neurological Disorders and Stroke [Grant NS107671] to GWA https://doi.org/10.1124/jpet.119.263350.

S This article has supplemental material available at jpet.aspetjournals.org. and replace glucose as the predominant neuronal energy substrate (Morris, 2005) (Fig. 1A). This metabolic state, referred to as ketosis, has been convincingly demonstrated to reduce incidence of seizures, especially in children with refractory epilepsy (Kinsman et al., 1992; Freeman et al., 1998; Hassan et al., 1999; Freiman et al., 2006; Taub et al., 2014), but also in adults (Kinsman et al., 1992). A meta-analysis of seven studies of the classic ketogenic diet in both adolescents and adults found that the diet was associated with $>50 \%$ seizure reduction in one-half of the patients treated, and $13 \%$ of the responsive patients were seizure free (Payne et al., 2011). Fasting produces similar metabolic shifts and its antiepileptic properties have been recognized for millennia (Hartman et al., 2013).

Despite the therapeutic importance of the ketogenic diet, the mechanisms by which it reduces seizures are still unclear. The ketone bodies produced by a ketogenic diet have received most attention in studies investigating the anticonvulsant action of this diet, and in particular, $\beta$-hydroxybutyrate (BHB), a ketone body that accumulates during ketosis (Fig. 1A). The antiepileptic efficacy of ketogenic diets has been positively correlated with BHB reaching an optimal serum concentration of $4 \mathrm{mM}$ (Gilbert et al., 2000). Several potentially therapeutic effects have been attributed to BHB, including replacement of glucose as a neuronal energy source 
A

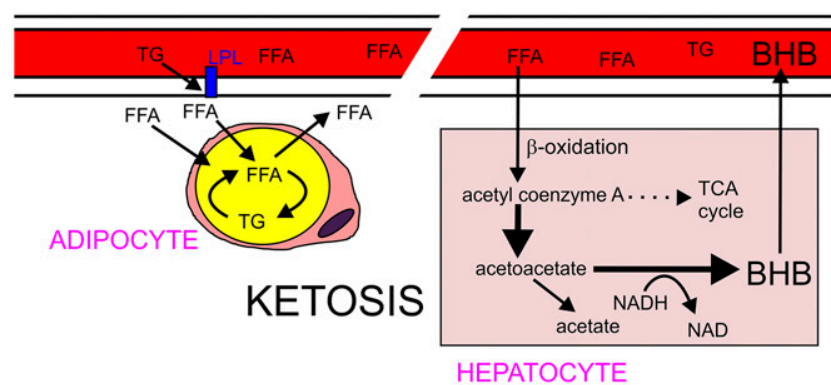

B

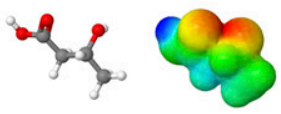

$\beta$-hydroxybutyric acid (BHB)

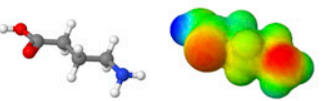

$\gamma$-aminobutyric acid (GABA)

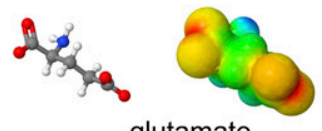

glutamate

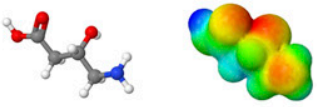

$\gamma$-amino- $\beta$-hydroxy butyric acid (GABOB)
C

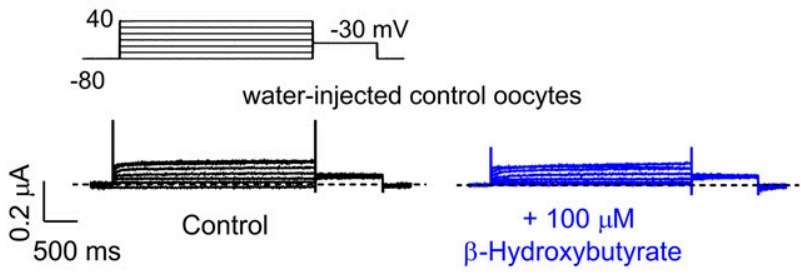

E

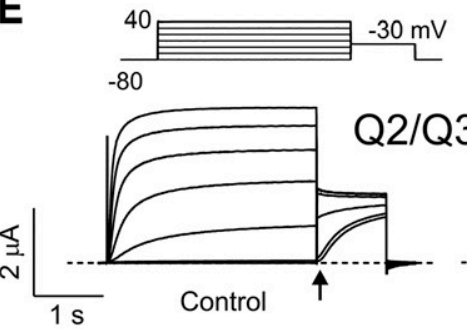

H

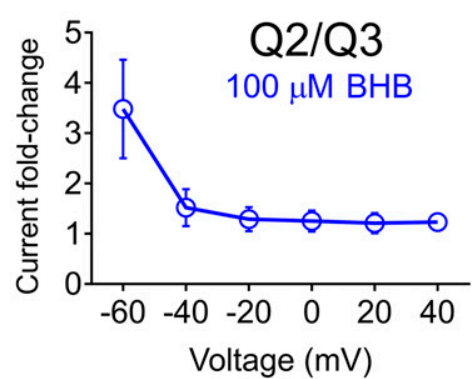

D

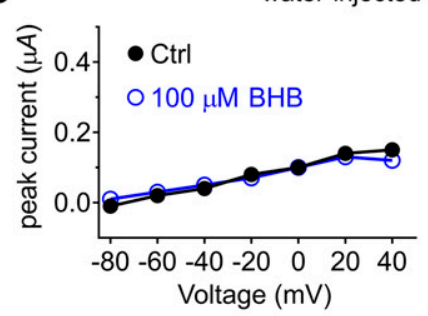

F

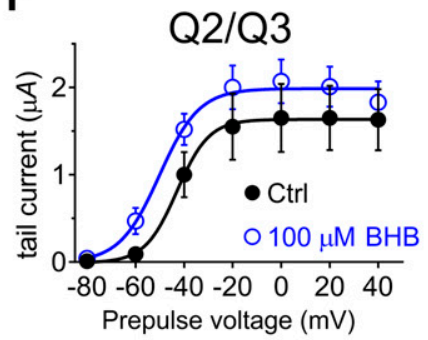

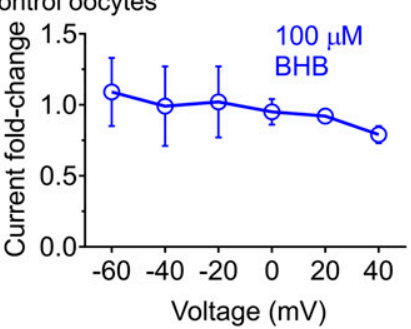

G

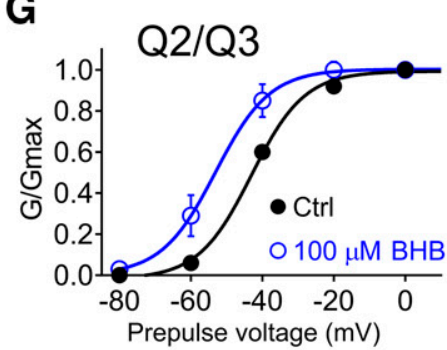

Fig. 1. BHB activates KCNQ2/3 potassium channels. (A) Schematic illustration of the generation of BHB during ketosis. FFA, free fatty acids; LPL, lipoprotein lipase; red areas, blood vessel; TCA, tricarboxylic acid cycle; TG, triglycerides. (B) Electrostatic surface potentials (red, electron dense; blue, electron poor; green, neutral) and structures calculated and plotted using Jmol. (C) TEVC recording of water-injected Xenopus laevis oocytes. Mean traces show no effect of BHB $(100 \mu \mathrm{M})$ on endogenous currents $(n=5)$. Dashed line here and throughout, zero current level. Upper inset, voltage protocol. (D) Left panel, mean I/V relationship from water-injected traces as in $(\mathrm{C})(n=5)$. Right panel, mean current fold change in response to $\mathrm{BHB}(100 \mu \mathrm{M})$ in waterinjected oocytes $(n=5)$. Error bars indicate S.E.M. (E-J) TEVC recordings of KCNQ2/3 expressed in Xenopus oocytes in the absence (control) or presence of BHB $(n=5)$. Dashed line here and throughout, zero current level. Voltage protocol as in (C). Error bars indicate S.E.M. (E) Mean traces. (F) Mean tail current vs. prepulse voltage [recorded here and throughout at arrow in (E)]. (G) Normalized tail currents [conductance/maximal conductance $\left(G / G_{\text {max }}\right)$; recorded here and throughout at arrow in (E)] vs. prepulse voltage. (H) Voltage dependence of BHB augmentation of KCNQ2/3 activity. (I) Dose-dependent fold increase in current at $-60 \mathrm{mV}$ in response to BHB. $(J)$ Dose-dependent changes in resting membrane potential $\left(E_{\mathrm{M}}\right)$ in response to BHB.

(LaManna et al., 2009), as well as also less intuitive actions. These include activation of hydroxyl-carbolic acid receptor 2 by $\mathrm{BHB}$, which may be neuroprotective via prostaglandin $\mathrm{D}_{2}$ production (Rahman et al., 2014); BHB also facilitates synaptic vesicle recycling (Hrynevich et al., 2016). Thus, ketone bodies such as BHB may exert a spectrum of effects on neurons to quell aberrant hyperexcitability. A greater understanding of the mechanisms underlying the ketogenic diet may lead to molecular intervention that mimics the beneficial effects of ketosis without the associated side effects, which can include gastrointestinal disruption and vitamin and mineral deficiencies (Hahn et al., 1979; Tallian et al., 1998; Bergqvist et al., 2003).

$\mathrm{K}^{+}$efflux is the primary force behind the cellular repolarization that ends action potentials, and is also instrumental in setting and maintaining resting membrane potential and preventing neuronal hyperexcitability. In metazoan nervous systems, KCNQ2-5 channels generate the M-current (Biervert et al., 1998; Singh et al., 1998), which is a muscarinic-inhibited voltage-gated potassium $\left(\mathrm{K}_{\mathrm{v}}\right)$ current (Brown and Adams, 1980) that plays an essential part in regulating neuronal excitability (Wang et al., 1998). The M-current is thought to be 
generated primarily by KCNQ2 and KCNQ3 subunits in the form of heteromeric (KCNQ2/3) channels (Wang et al., 1998, 2009). KCNQ2/3 channels do not inactivate, and open at relatively hyperpolarized membrane potentials. Therefore, they are uniquely evolved to regulate neuronal excitability, and accordingly their gating state governs phasic versus tonic firing of neurons (Wang et al., 1998). Furthermore, genetic disruption of KCNQ2 or KCNQ3 function causes epilepsy in humans and mice (Biervert et al., 1998; Schroeder et al., 1998; Singh et al., 1998, 2003, 2008; Watanabe et al., 2000; Soh et al., 2014). We recently discovered that GABA, the primary inhibitory neurotransmitter, can directly activate specific members of the KCNQ $\left(\mathrm{K}_{\mathrm{v}} 7\right)$ family of $\mathrm{K}_{\mathrm{v}}$ channels (Manville et al., 2018). In the same study, we found that two naturally occurring GABA analogs, $\gamma$-amino- $\beta$-hydroxybutyric acid (GABOB) and the ketone body $\mathrm{BHB}$, can also activate KCNQ2/3 channels. Since KCNQ2/3 activation is inhibitory to neuronal activity, and is the mechanism of action for the class of anticonvulsants including retigabine (Rundfeldt, 1999; Main et al., 2000; Wickenden et al., 2000), here we sought to determine the underlying molecular mechanisms and potential anticonvulsant significance of $\mathrm{BHB}$ activation of $\mathrm{KCNQ} 2 / 3$. Our results suggest an important role for KCNQ2/3 activation in BHB anticonvulsant activity.

\section{Materials and Methods}

Pentylene Tetrazole Chemoconvulsant Assay. The anticonvulsant activities of test compounds were compared in male C57BL/6 mice (Charles River Laboratories) aged 2 to 3 months. Mice were housed and used according to the recommendations in the National Institutes of Health's Guide for the Care and Use of Laboratory Animals (National Institutes of Health publication, eighth edition, 2011). The study was approved by the Institutional Animal Care and Use Committee of the University of California, Irvine. Chemicals were sourced from Sigma (St. Louis, MO) unless otherwise indicated. A pentylene tetrazole (PTZ) chemoconvulsant assay was used, as we previously described (Abbott et al., 2014). BHB and GABOB were diluted in Hanks' balanced salt solution for injection. Mice were injected intraperitoneally with GABOB and/or BHB, or Hanks' balanced salt solution vehicle control, and then 30 minutes later were injected intraperitoneally with $80 \mathrm{mg} / \mathrm{kg}$ PTZ. Following the PTZ injection, mice were caged individually and the observer (G.W.A.), blinded to the drug used, recorded (over 20 minutes) the seizure latencies (time to first tail flick or seizure), clonic and tonic incidence, and mortality.

Channel Subunit Complementary RNA Preparation and Xenopus laevis Oocyte Injection. Complementary RNA (cRNA) transcripts encoding human KCNQ2 or KCNQ3 were generated by in vitro transcription using the polymerase mMESSAGE mMACHINE T7 Kit (Thermo Fisher Scientific), after vector linearization, from cDNA subcloned into plasmids incorporating Xenopus laevis $\beta$-globin $5^{\prime}$ and $3^{\prime}$ untranslated regions flanking the coding region to enhance translation and cRNA stability. cRNA was quantified by spectrophotometry. Mutant channel cDNAs were generated by sitedirected mutagenesis using a QuikChange kit according to manufacturer's protocol (Stratagene, San Diego, CA) and then corresponding cRNAs were prepared as previously noted. Defolliculated stage V and VI Xenopus laevis oocytes (Ecocyte Bioscience, Austin, TX) were injected with $\mathrm{K}_{\mathrm{v}}$ channel $\alpha$ subunit cRNAs (5 ng). Oocytes were incubated at $16^{\circ} \mathrm{C}$ in Barth's saline solution (Ecocyte Bioscience) containing penicillin and streptomycin, with daily washing, for 3-5 days prior to twoelectrode voltage-clamp (TEVC) recording.

Two-Electrode Voltage-Clamp Recording. TEVC recording was performed at room temperature with an OC-725C amplifier (Warner Instruments, Hamden, CT) and pClamp8 software (Molecular
Devices, Sunnyvale, CA) 3-5 days after cRNA injection as described in the previous section. Oocytes were placed in a small-volume oocyte bath (Warner Instruments) and viewed with a dissection microscope. Chemicals were sourced from Sigma. The bath solution contained the following: $96 \mathrm{mM} \mathrm{NaCl}, 4 \mathrm{mM} \mathrm{KCl}, 1 \mathrm{mM} \mathrm{MgCl}_{2}, 1 \mathrm{mM} \mathrm{CaCl}_{2}$, and $10 \mathrm{mM}$ HEPES ( $\mathrm{pH} 7.6)$. BHB was stored at $4^{\circ} \mathrm{C}$ as a $480 \mathrm{mM}$ stock in $100 \%$ ethanol and diluted to working concentrations on each experimental day. GABOB was stored at $-80^{\circ} \mathrm{C}$ as $1 \mathrm{M}$ stocks in molecular grade $\mathrm{H}_{2} \mathrm{O}$ and diluted to working concentrations on each experimental day. All compounds were introduced to the recording bath via gravity perfusion at a constant flow of $1 \mathrm{ml} / \mathrm{min}$ for 3 minutes prior to recording. Pipettes were of 1 to $2 \mathrm{M} \Omega$ resistance when filled with $3 \mathrm{M} \mathrm{KCl}$. Currents were recorded in response to pulses between -80 and +40 at $20 \mathrm{mV}$ intervals, or a single pulse to $+40 \mathrm{mV}$, from a holding potential of -80 $\mathrm{mV}$, to yield current-voltage relationships and current magnitude, and to quantify the activation rate. TEVC data analysis was performed with Clampfit (Molecular Devices) and Graphpad Prism software (GraphPad, San Diego, CA); values are stated as mean \pm S.E.M. Normalized tail currents were plotted versus prepulse voltage and fitted with a single Boltzmann function:

$$
g=\frac{\left(A_{1}-A_{2}\right)}{\left\{1+\exp \left[V_{1 / 2}-\left(V / V_{\mathrm{s}}\right)\right]\right\} y+A_{2}}
$$

where $g$ is the normalized tail conductance; $A_{1}$ is the initial value at $\infty ; A_{2}$ is the final value at $+\infty ; V_{1 / 2}$ is the half-maximal voltage of activation; and $V_{\mathrm{s}}$ is the slope factor. Activation and deactivation kinetics were fitted with single exponential functions.

Chemical Structures, In Silico Docking, and Sequence Analyses. Chemical structures and electrostatic surface potentials (range $=-0.1$ to 0.1 ) were plotted using Jmol, an open-source Java viewer for chemical structures in three dimensions (http://jmol.org/). For docking, the Xenopus laevis KCNQ1 cryogenic electron microscopy structure (Sun and MacKinnon, 2017) was first altered to incorporate KCNQ3 residues known to be important for retigabine binding, followed by energy minimization using the GROMOS 43B1 force field (van Gunsteren, 1996), in DeepView (Johansson et al., 2012), as we previously described (Manville et al., 2018). Thus, Xenopus laevis KCNQ1 amino acid sequence LITTLYIGF was converted to LITAWYIGF, the underlined W being W265 in human KCNQ3. In addition, $\bar{X}$ enopus laevis KCNQ1 sequence WWGVVTVTTIGYGD was converted to WWGLITLATIGYGD, the underlined L being Leu314 in human KCNQ3. The surrounding nonmutated sequences are shown to illustrate the otherwise high sequence identity in these stretches. Unguided docking of $\mathrm{BHB}, \mathrm{GABOB}$, and retigabine to predict native binding sites was performed using SwissDock (Grosdidier et al., 2011b) with CHARMM force fields (Grosdidier et al., 2011a).

Statistical Analysis. All values are expressed as mean \pm S.E.M. Anticonvulsant actions were compared using a two-tailed $t$ test followed by Bonferroni correction, except for mortality which was compared using $\chi^{2}$ analysis. Statistical significance was defined as $P<0.05$.

\section{Results}

BHB Activates KCNQ2/3 Channels to Hyperpolarize Cell Membrane Potential. Similar to the anticonvulsant and KCNQ2/3 activator retigabine (Kim et al., 2015), GABA and BHB both harbor strongly negative electrostatic surface potential localized close to a carbonyl oxygen; in contrast, glutamate, an excitatory neurotransmitter, does not (Fig. 1B), and neither does it activate KCNQ2/3 channels (Manville et al., 2018). Here, we used TEVC electrophysiology in the Xenopus laevis oocyte expression system, which permits longterm recording of ion channel activity (expressed after injection of channel subunit cRNA) and large currents with little baseline drift, to determine the molecular mechanisms underlying BHB enhancement of KCNQ2/3 channels. First, 
water-injected control oocytes did not respond to bath-applied BHB (100 $\mu \mathrm{M})$, verifying oocytes as a suitable system for mechanistic studies (Fig. 1, C and D). In contrast, BHB (100 $\mu \mathrm{M})$ augmented currents generated by coinjection of cRNA encoding KCNQ2 and KCNQ3, which produces heteromeric KCNQ2/3 channels. This effect was readily apparent in the tail currents, which permit quantification of activation by various prepulse potentials, measured at a single tail potential (in this case, $-30 \mathrm{mV}$ ) to eliminate effects of driving force and give a comparison of the voltage dependence and fraction of channels opened (Fig. 1, E and F). BHB (100 $\mu \mathrm{M})$ increased KCNQ2/3 tail currents by $\sim 0.5 \mu \mathrm{A}$ between membrane potentials of -60 to $+20 \mathrm{mV}$, with lesser absolute increases at -80 and $+40 \mathrm{mV}$ (Fig. $1 \mathrm{~F}$ ). The relatively uniform absolute increase in current from -60 to $+20 \mathrm{mV}$ had the effect of negative shifting the midpoint voltage dependence of KCNQ2/ 3 activation by $-10 \mathrm{mV}$ (from $-43.1 \pm 1.4$ to $-53.1 \pm 1.7 \mathrm{mV}$; $n=6, P=0.001$ ) (Fig. 1G; Supplemental Fig. 1; Supplemental Table 1). The strongest augmentation in terms of fold increase versus baseline current occurred at $-60 \mathrm{mV}$, a subthreshold potential at which $\mathrm{K}_{\mathrm{v}}$ channel activity is especially influential in governing cellular excitability (Fig. 1H). Accordingly, BHB produced a maximal 3.5-fold mean increase in KCNQ2/3 current at $-60 \mathrm{mV}\left(\mathrm{EC}_{50}=0.7 \mu \mathrm{M}\right)$ (Fig. 1I), resulting in a resting membrane potential hyperpolarization of $-18 \mathrm{mV}$ at optimal BHB doses (Fig. 1J).

BHB Activates KCNQ3 but Not KCNQ2 Potassium Channels. We next tested KCNQ2 and KCNQ3 isoforms individually to determine whether one or both responded to BHB. Measuring the BHB sensitivity of homomeric KCNQ2 channels using a similar approach to that in Fig. 1, we found that they were insensitive to $\mathrm{BHB}$, even up to $1 \mathrm{mM}$ [voltage dependence of activation $\left(V_{0.5 a c t i v a t i o n}\right)$ was $-43.2 \pm 0.7 \mathrm{mV}$ in the absence of BHB vs. $-44.6 \pm 0.7 \mathrm{mV}$ with $\mathrm{BHB} ; n=5$, $P=0.2$ ] (Fig. 2; Supplemental Table 2).

In contrast, $\mathrm{BHB}$ produced robust augmentation of currents generated by KCNQ3* (an expression-optimized KCNQ3A315T mutant that facilitates accurate analysis of homomeric KCNQ3 currents) (Zaika et al., 2008) (Fig. 3, A and B) and negative-shifted the $V_{0.5 a c t i v a t i o n}($ by $-11 \mathrm{mV}$ with $100 \mu \mathrm{M}$ BHB; from $-42.2 \pm 0.9$ to $-53.2 \pm 1.0 \mathrm{mV} ; n=6, P=1.0 \times$ $10^{-5}$ ) (Fig. 3, B and C). This produced a $>8.5$-fold mean increase in current at $-60 \mathrm{mV}$ with $100 \mu \mathrm{M} \mathrm{BHB}\left(\mathrm{EC}_{50}=1.4\right.$ $\pm 0.5 \mu \mathrm{M}$ BHB) (Fig. 3D), and lesser effects at more positive voltages (Fig. 3E; Supplemental Fig. 2; Supplemental Table 3). Thus, BHB activation of KCNQ channels exhibits isoform specificity and in KCNQ2/3 channels must be mediated by KCNQ3. The high sensitivity of KCNQ3 for BHB sharply contrasts with canonical $\mathrm{GABA}_{\mathrm{A}}$ receptors, which are insensitive to BHB up to at least $5 \mathrm{mM}$ (Yang et al., 2007). Moreover, it places the BHB sensitivity of KCNQ3 channels (and KCNQ2/3 channels) well within the clinical range observed in the ketogenic diet; for example, previous studies found that occipital lobe $\beta$-hydroxybutyrate concentrations in children rose from $\sim 50 \mu \mathrm{M}( \pm 50 \mu \mathrm{M})$ to $\sim 1 \mathrm{mM}$ after 3 days of fasting (Pan et al., 2000).

BHB Directly Activates KCNQ3 via the S5 Tryptophan W265. KCNQ channels are canonical $\mathrm{K}_{\mathrm{v}}$ channels, formed from tetramers of $\alpha$ subunits each bearing six transmembrane segments (S1-S6). Segments S1-S4 form the voltage-sensing domain; segments S5 and S6 form the pore module with the selectivity filter through which $\mathrm{K}^{+}$ions pass lying between them and dipping midway into the plasma membrane from the extracellular side (Fig. 4A). KCNQ2-5 subunits, but not KCNQ1, each contain a tryptophan in the S5 segment (W265 in KCNQ3) (Fig. 4A) that mediates channel activation by retigabine and related anticonvulsants. We previously found that activation by GABA requires this residue, and that it is important for direct binding of GABA (Manville et al., 2018). In silico binding studies predict that, like retigabine, BHB can bind at or close to KCNQ3W265 (Fig. 4, B and C). Furthermore, in a structural model of KCNQ3 produced by substitution of key residues in the cryogenic electron microscopy structure of Xenopus KCNQ1, W265 lies in a crevice apparently accessible from the extracellular face by BHB and similar-sized molecules (Fig. 4D). In support of this, we previously found that extracellularly applied ${ }^{3} \mathrm{H}$-GABA, which does not readily cross the plasma membrane, bound to Xenopus oocytes expressing KCNQ2-5, but not noninjected oocytes or those expressing KCNQ1, which lack the W265 equivalent (Manville et al., 2018). To test the hypothesis that W265 mediates BHB activation of KCNQ3, we expressed mutant KCNQ2/3 channels with leucine substituted for the S5 tryptophan and found that they were largely insensitive even up to $4 \mathrm{mM} \mathrm{BHB}$, except for a small run-up at more positive voltages (Fig. 4E). Thus, BHB
A

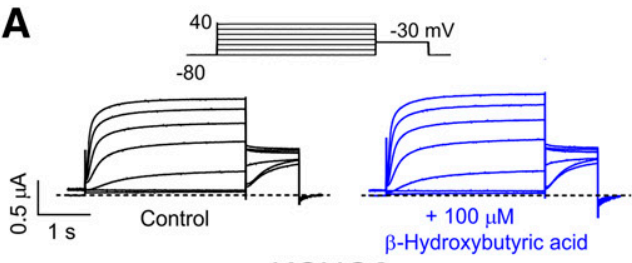
KCNQ2

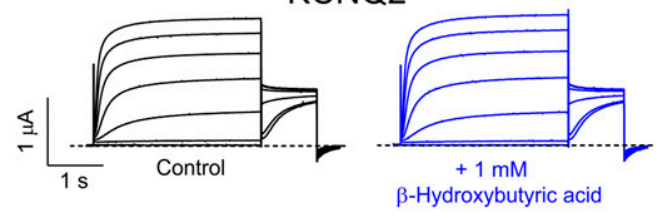

B
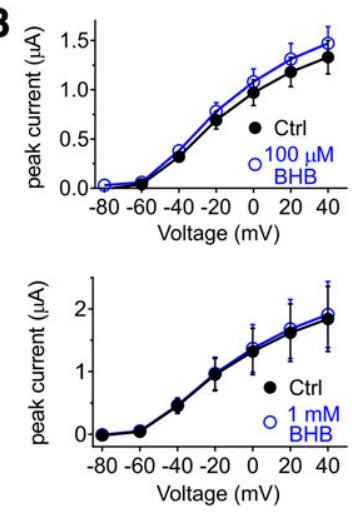
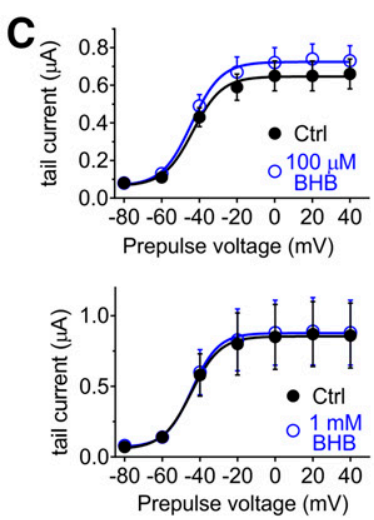
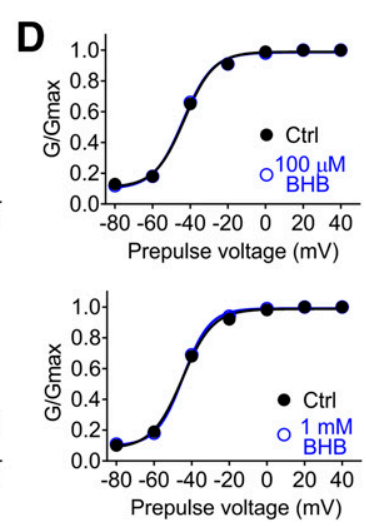

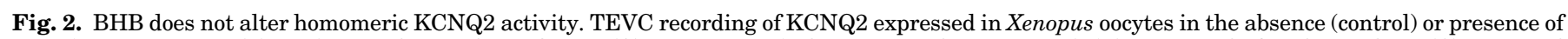

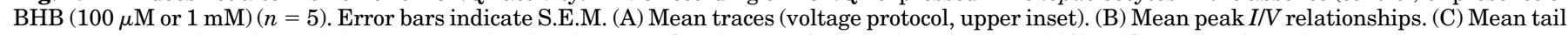
current vs. prepulse voltage. (D) Mean normalized tail current [conductance/maximal conductance $\left(G / G_{\text {max }}\right)$ ] vs. prepulse voltage. 
A

\section{Control}
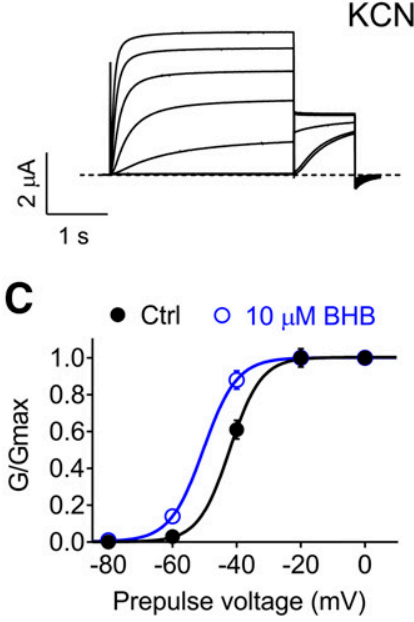

$100 \mu \mathrm{M}$ BHB
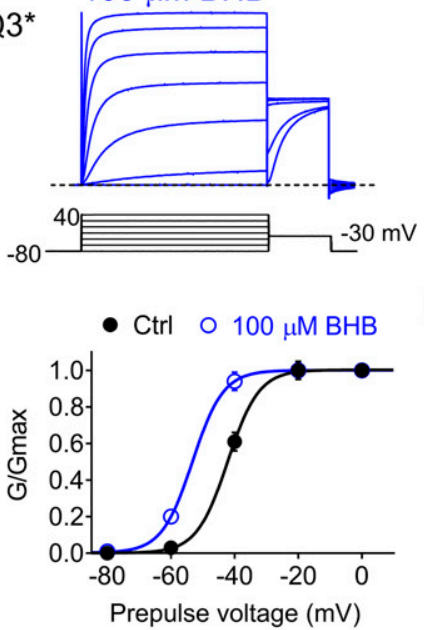

B

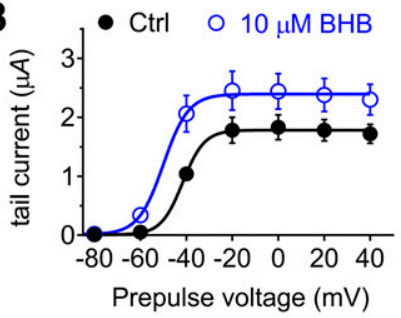

D

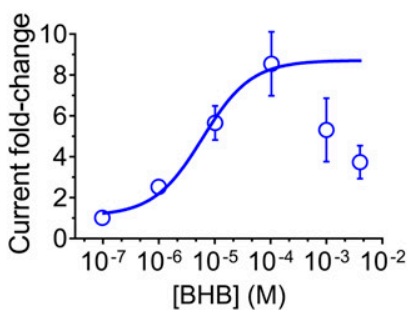

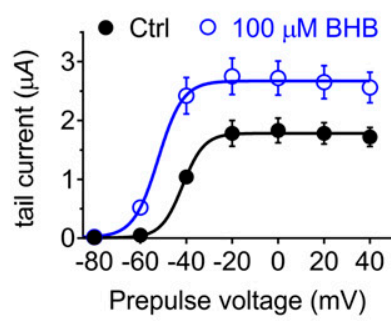

E

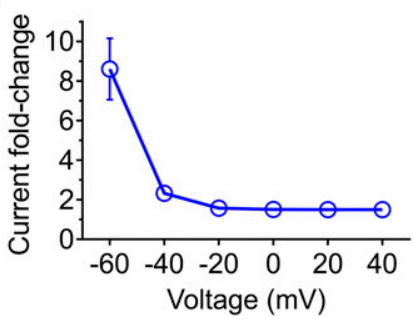

Fig. 3. BHB activates homomeric KCNQ3* potassium channels. TEVC recording of KCNQ3* expressed in Xenopus oocytes in the absence (control) or presence of BHB $(10 \mathrm{nM}-10 \mathrm{mM})(n=6)$. Error bars indicate S.E.M. (A) Mean traces (voltage protocol, upper inset). (B) Mean tail current vs. prepulse voltage. (C) Mean normalized tail current [conductance/maximal conductance $\left(G / G_{\max }\right)$ ] vs. prepulse voltage. (D) Dose response of BHB vs. KCNQ3* current fold increase at $-60 \mathrm{mV}$. (E) Voltage dependence of BHB effects on KCNQ3* activity.

did not negative shift the $V_{0.5 a c t i v a t i o n}$ of W236L-KCNQ2/ W265L-KCNQ3 channels, even causing a positive shift at 4 $\mathrm{mM}$, from $-59.5 \pm 0.5$ to $-55.7 \pm 0.7 \mathrm{mV} ; n=5, P=0.003$ (Fig. 4F); therefore, it did not increase the current at the physiologically important subthreshold membrane potential of $-60 \mathrm{mV}$ (Fig. 4G; Supplemental Fig. 3; Supplemental Table 4).

Partial KCNQ2/3 Agonist GABOB Diminishes BHB Activation of KCNQ2/3. To further support the premise that BHB activates KCNQ2/3 channels by binding to KCNQ3W265, we used a competition assay. We previously found that GABOB, a naturally occurring GABA analog and lesser-known neurotransmitter, activates KCNQ2/3 channels with high affinity $\left(\mathrm{EC}_{50}=0.12 \mu \mathrm{M}\right.$ at $\left.-60 \mathrm{mV}\right)$ but lower efficacy than GABA or BHB. Thus, GABOB acts as a partial KCNQ2/3 agonist. In silico binding studies predict that GABOB also binds to KCNQ3-W265 and that it can access W265 from the extracellular side (Fig. 5, A and B). Accordingly, we previously found that GABOB competes out GABA and even the synthetic drug retigabine, which also binds to W265, reducing their efficacy for KCNQ2/3 activation (Manville et al., 2018). Here, we employed a similar assay and found that GABOB $(100 \mu \mathrm{M})$ also greatly reduces the efficacy of $\mathrm{BHB}(10 \mu \mathrm{M})$, presumably by occupying the W265-associated
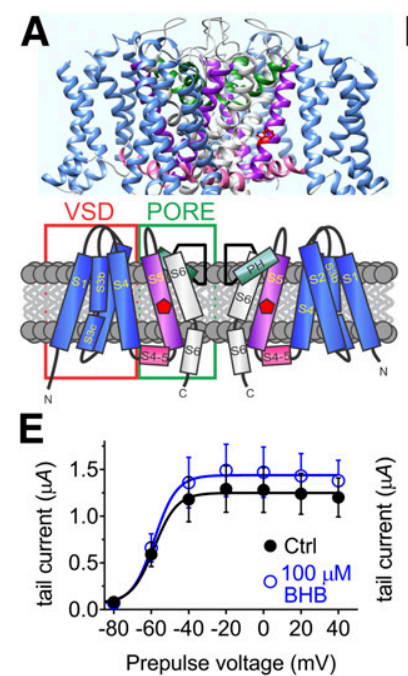
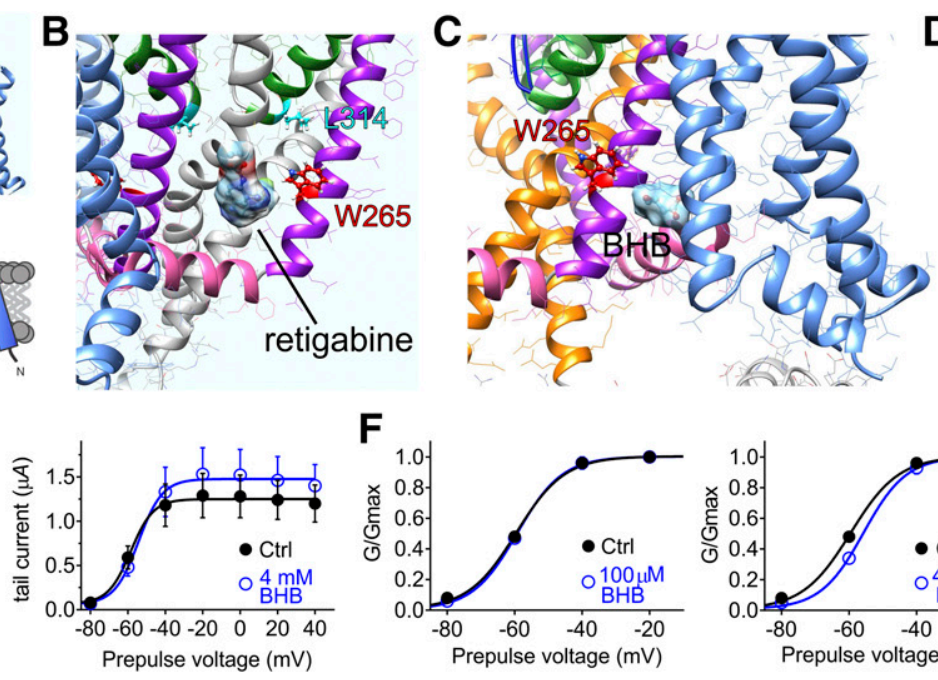

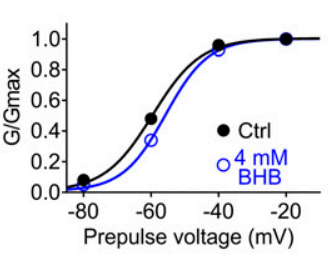

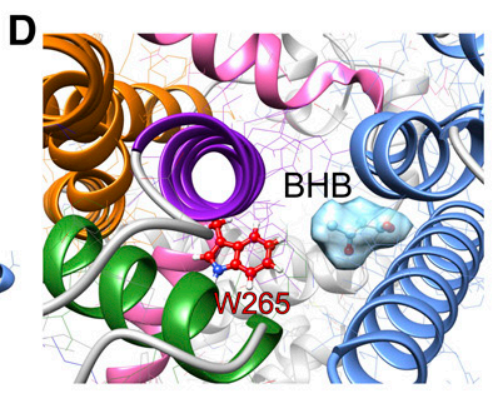

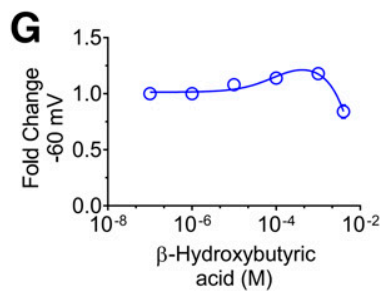

Fig. 4. BHB activation of KCNQ2/3 requires an S5 tryptophan. (A) Upper panel, chimeric KCNQ1/KCNQ3 structural model (red pentagon, KCNQ3W265); lower panel, schematic illustration showing membrane topology; domain coloring: blue, VSD; pink, S4-5 linker; purple, S5; light gray, S6. VSD, voltage-sensing domain; pentagon, KCNQ3-W265. (B) Close-up side view of KCNQ structure as in (A) showing results of SwissDock docking of retigabine in the structural model. (C) Close-up side view of KCNQ structure as in (A) showing results of SwissDock docking of BHB in the structural model. (D) Close-up view of KCNQ structure from extracellular face, showing results of SwissDock docking of BHB in the structural model. (E-G) TEVC recordings of Xenopus laevis oocytes showing effects of BHB (100 $\mu \mathrm{M}-4 \mathrm{mM})$ on heteromeric KCNQ2-W236L/KCNQ3-W265L channels; $n=6$; voltage protocol as in Fig. 3. Error bars indicate S.E.M. (E) Mean tail current vs. prepulse voltage. (F) Mean normalized tail current [conductance/maximal conductance $\left.\left(G / G_{\max }\right)\right]$ vs. prepulse voltage. $(\mathrm{G})$ Dose response of BHB vs. KCNQ2-W236L/KCNQ3-W265L current fold increase at $-60 \mathrm{mV}$. 
A
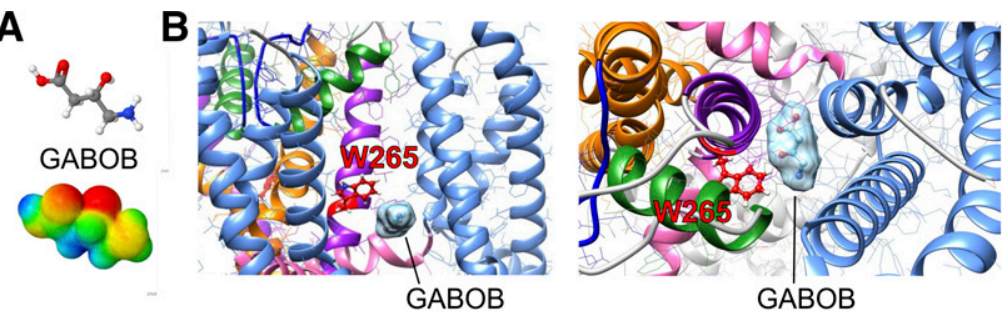

C
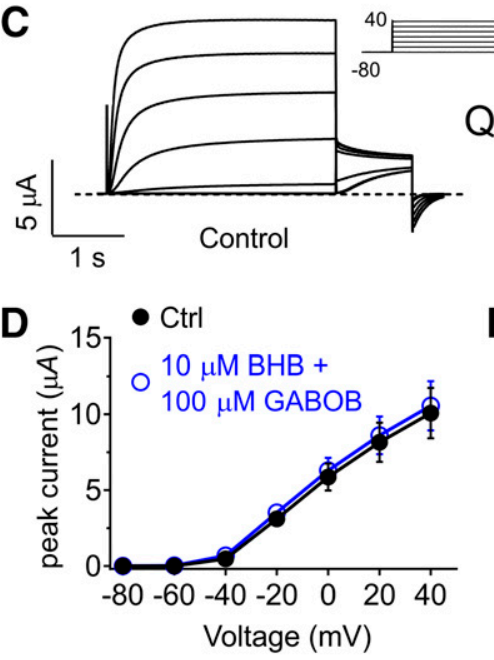

$\mathbf{F}$

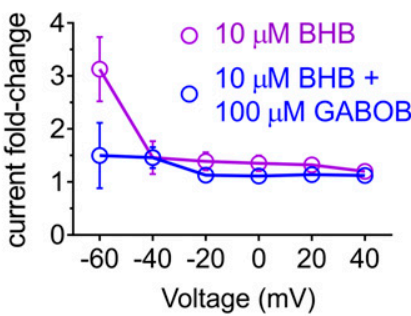

GABOB

\section{E}
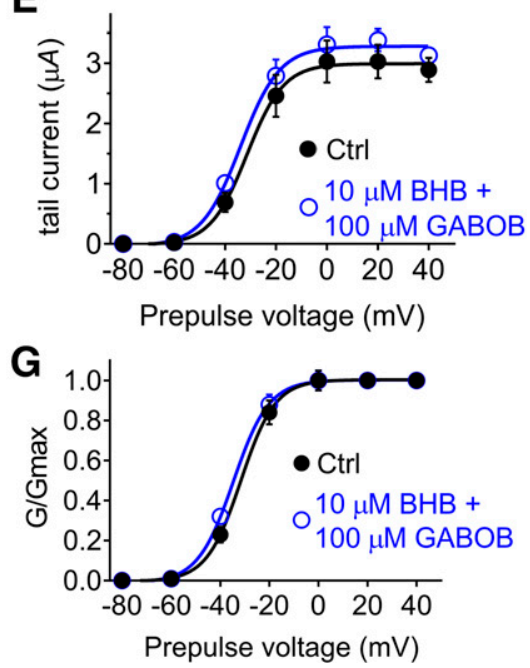

Fig. 5. GABOB diminishes $\mathrm{BHB}$ activation of $\mathrm{KCNQ} 2 / 3$. (A) GABOB electrostatic surface potential (red, electron dense; blue, electron poor; green, neutral) and structure calculated and plotted using Jmol. (B) Left panel, side view of KCNQ1/3 chimera model structure, showing results of SwissDock docking of GABOB; right panel, view of KCNQ1/3 chimera model structure from the extracellular face, showing results of SwissDock docking of GABOB. (C-G) TEVC recordings of Xenopus laevis oocytes showing effects of BHB $(10 \mu \mathrm{M})$ in the presence of GABOB $(100 \mu \mathrm{M})$ on heteromeric KCNQ2/3 channels; $n=5$. Error bars indicate S.E.M. (C) Mean traces (voltage protocol, upper inset). (D) Peak current. (E) Mean tail current vs. prepulse voltage. (F) Fold change in tail current vs. prepulse for $10 \mu \mathrm{M}$ BHB with $100 \mu \mathrm{M}$ GABOB vs. without $100 \mu \mathrm{M}$ GABOB. (G) Mean normalized tail current [conductance/maximal conductance $\left.\left(G / G_{\max }\right)\right]$ vs. prepulse voltage. binding site and disallowing $\mathrm{BHB}$ binding. Thus, in the presence of GABOB, BHB was unable to shift the KCNQ2/ $3 V_{0.5 a c t i v a t i o n}[-32.2 \pm 1.1$ (control) vs. $-34.8 \pm 1.0 \mathrm{mV}(\mathrm{BHB}+$ GABOB); $n=5, P=0.12$ ] (Fig. 5, C-G; Supplemental Table 5). In summary, electrophysiological, mutagenesis, and GABOB competition assays support the hypothesis that BHB activates KCNQ2/3 channels by binding to KCNQ3-W265.

BHB Anticonvulsant Activity Is Diminished by GABOB. The capacity of KCNQ2/3 partial agonist GABOB to competitively inhibit $\mathrm{BHB}$ binding to KCNQ3 facilitated a mechanistic analysis of the anticonvulsant effects of BHB. We first tested the efficacy of $\mathrm{BHB}$ as an anticonvulsant using the pentylene tetrazole chemoconvulsant assay in mice, intraperitoneally injecting BHB 30 minutes before intraperitoneal injection of $80 \mathrm{mg} / \mathrm{kg} \mathrm{PTZ}$, and then recording seizures and mortality in a blinded manner for the next 20 minutes. We found that BHB was highly effective at $200 \mathrm{mg} / \mathrm{kg}$, and less so at $40 \mathrm{mg} / \mathrm{kg}$, in increasing latency to first seizure (Fig. 6A), and decreasing clonic (Fig. 6B) and tonic (Fig. 6C) seizures, as well as increasing survival (Fig. 6D). Most strikingly, $200 \mathrm{mg} / \mathrm{kg}$ BHB completely prevented tonic seizures and seizure-related death, which each occurred in $>50 \%$ of mice pretreated with vehicle instead of $\mathrm{BHB}$ (Fig. 6, C and D). Importantly, $200 \mathrm{mg} / \mathrm{kg}$ BHB corresponds to $\sim 2 \mathrm{mM} \mathrm{BHB}$, comparable with the $4 \mathrm{mM}$ serum levels correlated with optimal ketogenic diet anticonvulsant activity in patients (Gilbert et al., 2000).
GABOB $(200 \mathrm{mg} / \mathrm{kg})$ was ineffective as an anticonvulsant, but also did not predispose to seizures, resulting in seizure and mortality incidence similar to those observed for vehicle controls (Fig. 6). These findings were consistent with previous observations that GABOB is a weak anticonvulsant (Chemello et al., 1980; García-Flores and Farías, 1997). We hypothesized that if $\mathrm{GABOB}$ outcompeted $\mathrm{BHB}$ for the KCNQ3 binding site, as a partial agonist it would reduce the anticonvulsant efficacy of BHB, and this proved to be the case. Thus, GABOB (200 mg/kg) eliminated the anticonvulsant effects of $\mathrm{BHB}$ with respect to seizure latency, incidence, and mortality (Fig. 6). These findings, together with the cellular data, support the hypothesis that KCNQ2/3 channel activation is an important component of the anticonvulsant activity of BHB.

\section{Discussion}

The ketone bodies produced by fasting or a ketogenic diet are widely considered to be the principal components responsible for the anticonvulsant effects of these diets. In addition, ketone bodies including $\mathrm{BHB}$ may mediate other neuroprotective and anti-inflammatory effects. By inhibiting glycolytic flux upstream of pyruvate kinase, BHB competes with glucose as an energy provider in the brain (Valente-Silva et al., 2015; Boison, 2017). By shifting ketones such as BHB 
A
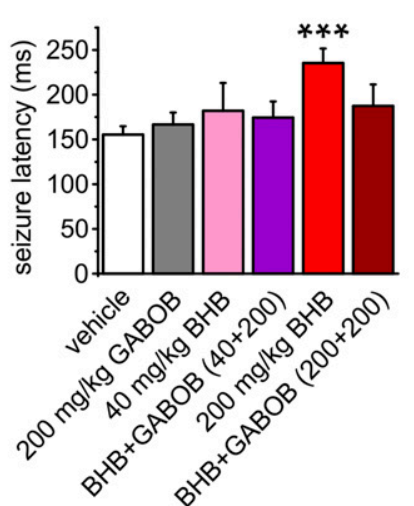

C

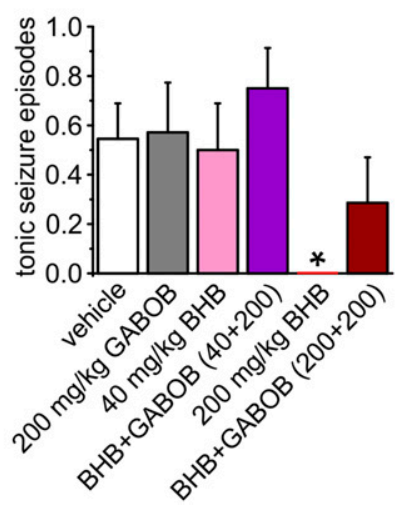

B
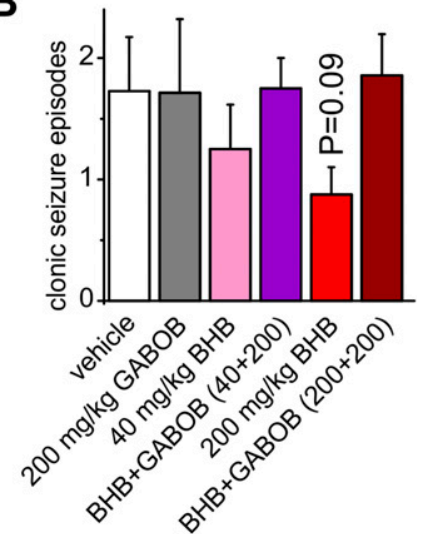

D

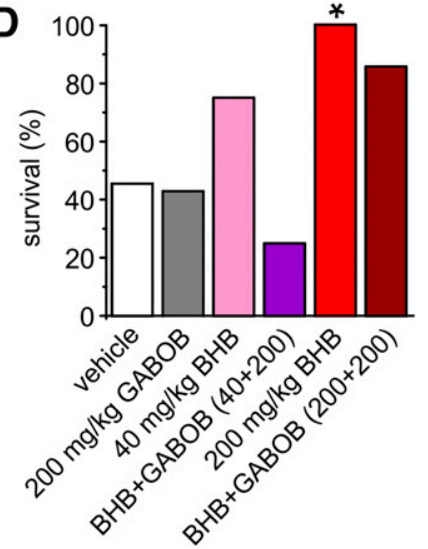

Fig. 6. GABOB diminishes anticonvulsant effects of BHB. Comparison of anticonvulsant effects of vehicle vs. 40 or $200 \mathrm{mg} / \mathrm{kg} \mathrm{BHB}$, in the absence $(n=8)$ or presence $(n=7-11)$ of $200 \mathrm{mg} / \mathrm{kg}$ GABOB, intraperitoneally injected 30 minutes before intraperitoneal injection of $80 \mathrm{mg} / \mathrm{kg}$ PTZ. Seizures were quantified during the first 20 minutes post-PTZ injection: (A) latency to first tail flick or seizure; *** $\mathrm{P}=0.001$ versus vehicle control. (B) clonic seizure episodes; (C) tonic seizure episodes; $* \mathrm{P}=0.02$ versus vehicle control. (D) seizure-related mortality; $* \mathrm{P}=0.02$ versus vehicle control. All other comparisons $\mathrm{P}>0.05$ versus vehicle control.

into oxidative metabolism, ketogenic diets also increase production of GABA (Zhang et al., 2015), itself an inhibitory neurotransmitter that we recently found activates KCNQ2/3 channels (Manville et al., 2018), in addition to its established role in activating canonical GABA receptors.

Canonical pentameric $\mathrm{GABA}_{\mathrm{A}}$ receptors, which are triggered to open when GABA binds to an extracellularly located, intersubunit GABA binding site, participate in either phasic or tonic inhibition, depending on their location and subunit composition (Kash et al., 2004; Belelli et al., 2009). Tonic extracellular GABA concentration in mammalian brain is calculated to be $0.16 \mu \mathrm{M}$ (Santhakumar et al., 2006; Lee et al., 2010), in contrast to the astronomical but transient peak GABA concentrations of several millimolars thought to occur in the synaptic cleft during neurotransmission (Scimemi, 2014).

The GABA $\mathrm{EC}_{50}$ value of $1 \mu \mathrm{M}$ we previously found for KCNQ2/3 channels endows them with equivalent sensitivity to that of the most sensitive $\alpha_{\mathrm{x}} \beta_{3} \gamma_{2} \mathrm{GABA}_{\mathrm{A}}$ receptors, which as a family exhibit a spectrum of sensitivities between 1 and $157 \mu \mathrm{M}$ (Böhme et al., 2004; Karim et al., 2013). In contrast, while KCNQ3 exhibited a BHB $\mathrm{EC}_{50}$ value of $1.4 \mu \mathrm{M}$ in the current study, even at 5-20 mM BHB was ineffective/weakly

effective as a $\mathrm{GABA}_{\mathrm{A}}$ receptor agonist (Yang et al., 2007). Thus, the complex, intersubunit GABA binding site of $\mathrm{GABA}_{\mathrm{A}}$ receptors provides much higher selectivity than the more permissive W265-based binding site of KCNQ3, which accepts GABA, BHB, and GABOB and likely other GABA analogs and metabolites with low or submicromolar affinities, and also binds larger molecules such as the synthetic anticonvulsant retigabine (Schenzer et al., 2005).

The reported ability of BHB to increase brain GABA levels could also point to a role for increased activity of canonical GABA receptors in the anticonvulsant action of the ketogenic diet due to downstream increases in GABA. However, our cellular electrophysiology and acute PTZ assay results support a hitherto unexpected, prominent, and direct role for BHB itself in activating KCNQ3-containing potassium channels, such as KCNQ2/3 heteromers. KCNQ2/3 channels are widely expressed in vertebrate neurons, where they play a prominent role in controlling neuronal excitability. KCNQ2/3 channels are open at subthreshold potentials and do not inactivate, but when inhibited by activation of muscarinic acetylcholine receptors, their closure permits neuronal firing. Presynaptic KCNQ2/3 channels are thought to modulate glutamate and GABA release, and are suggested to act pre- and postsynaptically to suppress neuronal excitability (Martire et al., 2004; Peretz et al., 2007). Therefore, KCNQ2/3 activation by BHB presents an attractive, novel, and plausible mechanism for the anticonvulsant effects of BHB.

We previously showed that GABOB is a partial agonist of KCNQ2/3 channels, with an $\mathrm{E}_{\mathrm{C} 50}$ value of $120 \mathrm{nM}$ (Manville et al., 2018). This is $>5$-fold more potent than BHB. Because GABOB has a much lower efficacy than BHB but higher potency, and each binds to the same residue, GABOB is able to prevent BHB binding at Trp265, but cannot activate KCNQ2/3 as effectively. We also previously showed that GABOB can reduce the efficacy of GABA and even retigabine with respect to KCNQ2/3 activation (Manville et al., 2018). In the present study, the lower dose of BHB we used in vivo was $40 \mathrm{mg} / \mathrm{kg}$, which is equivalent to $400 \mu \mathrm{M}$; this dose did not reduce seizures, whereas the higher dose of $200 \mathrm{mg} / \mathrm{kg}$ BHB $(2 \mathrm{mM})$ was effective against seizures (Fig. 6). GABOB was not effective against seizures at $200 \mathrm{mg} / \mathrm{kg}$ (1.7 mM) (Fig. 6). In the mouse model used herein, the lack of efficacy in preventing seizures of partial agonist GABOB is consistent with our prior findings, which showed that GABOB at $1 \mathrm{mM}$ is less efficacious with respect to KCNQ2/3 activation than $1 \mu \mathrm{M}$ BHB (Manville et al., 2018), a dose much lower than the lower dose of $400 \mu \mathrm{M}$ BHB that we found to be ineffective against seizures in the present study.

Vertebrate nervous systems contain many GABA analogs and metabolites, some of which, like GABA, also affect neuronal excitability, but unlike GABA do not modulate canonical GABA receptors and lack known targets. Our prior (Manville et al., 2018) and current studies suggest that W265 in human KCNQ3 evolved to act as a chemosensor for GABA and its analogs, providing an alternative mechanism for regulating neuronal excitability. We suggest that various neurotransmitters/metabolites including GABA, BHB, GABOB, and others compete for binding to KCNQ3-W265 and that the resultant activation $V_{1 / 2}$ of KCNQ3 and KCNQ2/3 channels is set by the specific profile of these molecules that the KCNQ3 subunits expressed at 
the cell surface are exposed to at any given time. Thus, if GABOB were prevalent, the cell would be more excitable than if BHB were prevalent. Since the affinity for KCNQ3 subunits of BHB is $\sim 10$-fold lower than that of GABOB, there would need to be an excess of BHB to outcompete GABOB for binding. Hence, even though the $\mathrm{KCNQ} \mathrm{EC}_{50}$ value for $\mathrm{BHB}$ is $\sim 1.4 \mu \mathrm{M}$ and maximal effects are observed at $100 \mu \mathrm{M}$, to overcome baseline GABOB levels BHB might need to be at a higher concentration, such as would be achieved during fasting or when on a ketogenic diet.

\section{Acknowledgments}

We are grateful to Lily Chen and Angele De Silva (University of California, Irvine) for generating mutant channel constructs.

\section{Authorship Contributions}

Participated in research design: Abbott.

Conducted experiments: Manville, Papanikolaou, Abbott.

Contributed new reagents or analytic tools: Abbott.

Performed data analysis: Manville, Abbott.

Wrote or contributed to the writing of the manuscript: Manville, Abbott.

\section{References}

Abbott GW, Tai KK, Neverisky DL, Hansler A, Hu Z, Roepke TK, Lerner DJ, Chen Q, Liu L, Zupan B, et al. (2014) KCNQ1, KCNE2, and $\mathrm{Na}^{+}$-coupled solute transporters form reciprocally regulating complexes that affect neuronal excitability. Sci Signal 7:ra22.

Belelli D, Harrison NL, Maguire J, Macdonald RL, Walker MC, and Cope DW (2009) Extrasynaptic GABAA receptors: form, pharmacology, and function. $J$ Neurosci 29 12757-12763.

Bergqvist AG, Chee CM, Lutchka L, Rychik J, and Stallings VA (2003) Selenium deficiency associated with cardiomyopathy: a complication of the ketogenic diet. Epilepsia 44:618-620.

Biervert C, Schroeder BC, Kubisch C, Berkovic SF, Propping P, Jentsch TJ, and Steinlein OK (1998) A potassium channel mutation in neonatal human epilepsy. Science 279:403-406.

Böhme I, Rabe H, and Lüddens H (2004) Four amino acids in the $\alpha$ subunits determine the $\gamma$-aminobutyric acid sensitivities of $\mathrm{GABA}_{\mathrm{A}}$ receptor subtypes. J Biol Chem 279:35193-35200.

Boison D (2017) New insights into the mechanisms of the ketogenic diet. Curr Opin Neurol 30:187-192.

Brown DA and Adams PR (1980) Muscarinic suppression of a novel voltage-sensitive $\mathrm{K}^{+}$current in a vertebrate neurone. Nature 283:673-676.

Chemello R, Giaretta D, Pellegrini A, and Testa G (1980) [Effect of gamma-aminobeta-hydroxybutyric acid (GABHB) on experimentally-induced epileptic activity] Riv Neurol 50:253-268.

Falco-Walter JJ, Scheffer IE, and Fisher RS (2018) The new definition and classification of seizures and epilepsy. Epilepsy Res 139:73-79.

Freeman JM, Vining EP, Pillas DJ, Pyzik PL, Casey JC, and Kelly LM (1998) The efficacy of the ketogenic diet-1998: a prospective evaluation of intervention in 150 children. Pediatrics 102:1358-1363.

Freiman TM, Surges R, Kukolja J, Heinemeyer J, Klar M, van Velthoven V, and Zentner $\mathrm{J}(2006) \mathrm{K}^{+}$-evoked $\left[{ }^{3} \mathrm{H}\right]$-norepinephrine release in human brain slices from epileptic and non-epileptic patients is differentially modulated by gabapentin and pinacidil. Neurosci Res 55:204-210.

García-Flores E and Farías R (1997) $\gamma$-Amino- $\beta$-hydroxybutyric acid as add-on therapy in adult patients with severe focal epilepsy. Stereotact Funct Neurosurg 69 : 243-246.

Gilbert DL, Pyzik PL, and Freeman JM (2000) The ketogenic diet: seizure control correlates better with serum $\beta$-hydroxybutyrate than with urine ketones. $J$ Child Neurol 15:787-790.

Grosdidier A, Zoete V, and Michielin O (2011a) Fast docking using the CHARMM force field with EADock DSS. J Comput Chem 32:2149-2159.

Grosdidier A, Zoete V, and Michielin O (2011b) SwissDock, a protein-small molecule docking web service based on EADock DSS. Nucleic Acids Res 39:W270-W277.

Hahn TJ, Halstead LR, and DeVivo DC (1979) Disordered mineral metabolism produced by ketogenic diet therapy. Calcif Tissue Int 28:17-22.

Hartman AL, Rubenstein JE, and Kossoff EH (2013) Intermittent fasting: a "new" historical strategy for controlling seizures? Epilepsy Res 104:275-279.

Hassan AM, Keene DL, Whiting SE, Jacob PJ, Champagne JR, and Humphreys P (1999) Ketogenic diet in the treatment of refractory epilepsy in childhood. Pediatr Neurol 21:548-552.

Hrynevich SV, Waseem TV, Hébert A, Pellerin L, and Fedorovich SV (2016) $\beta$-Hydroxybutyrate supports synaptic vesicle cycling but reduces endocytosis and exocytosis in rat brain synaptosomes. Neurochem Int 93:73-81.

Johansson MU, Zoete V, Michielin O, and Guex N (2012) Defining and searching for structural motifs using DeepView/Swiss-PdbViewer. BMC Bioinformatics 13:173.

Karim N, Wellendorph P, Absalom N, Johnston GA, Hanrahan JR, and Chebib M (2013) Potency of GABA at human recombinant $\mathrm{GABA}_{\mathrm{A}}$ receptors expressed in Xenopus oocytes: a mini review. Amino Acids 44:1139-1149.
Kash TL, Trudell JR, and Harrison NL (2004) Structural elements involved in activation of the $\gamma$-aminobutyric acid type A (GABAA) receptor. Biochem Soc Trans 32:540-546.

Kim RY, Yau MC, Galpin JD, Seebohm G, Ahern CA, Pless SA, and Kurata HT (2015) Atomic basis for therapeutic activation of neuronal potassium channels. Nat Commun 6:8116.

Kinsman SL, Vining EP, Quaskey SA, Mellits D, and Freeman JM (1992) Efficacy of the ketogenic diet for intractable seizure disorders: review of 58 cases. Epilepsia 33:1132-1136.

LaManna JC, Salem N, Puchowicz M, Erokwu B, Koppaka S, Flask C, and Lee Z (2009) Ketones suppress brain glucose consumption. Adv Exp Med Biol 645: 301-306.

Lee S, Yoon BE, Berglund K, Oh SJ, Park H, Shin HS, Augustine GJ, and Lee CJ (2010) Channel-mediated tonic GABA release from glia. Science 330:790-796.

Main MJ, Cryan JE, Dupere JR, Cox B, Clare JJ, and Burbidge SA (2000) Modulation of KCNQ2/3 potassium channels by the novel anticonvulsant retigabine. $\mathrm{Mol}$ Pharmacol 58:253-262.

Manville RW, Papanikolaou M, and Abbott GW (2018) Direct neurotransmitter activation of voltage-gated potassium channels. Nat Commun 9:1847.

Martire M, Castaldo P, D’Amico M, Preziosi P, Annunziato L, and Taglialatela M (2004) M channels containing KCNQ2 subunits modulate norepinephrine, aspartate, and GABA release from hippocampal nerve terminals. J Neurosci 24:592-597. Morris AA (2005) Cerebral ketone body metabolism. J Inherit Metab Dis 28:109-121.

Nordli DR Jr (2009) The ketogenic diet, four score and seven years later. Nat Clin Pract Neurol 5:12-13.

Pan JW, Rothman TL, Behar KL, Stein DT, and Hetherington HP (2000) Human brain $\beta$-hydroxybutyrate and lactate increase in fasting-induced ketosis. J Cereb Blood Flow Metab 20:1502-1507.

Payne NE, Cross JH, Sander JW, and Sisodiya SM (2011) The ketogenic and related diets in adolescents and adults-a review. Epilepsia 52:1941-1948.

Peretz A, Sheinin A, Yue C, Degani-Katzav N, Gibor G, Nachman R, Gopin A, Tam E, Shabat D, Yaari Y, et al. (2007) Pre- and postsynaptic activation of M-channels by a novel opener dampens neuronal firing and transmitter release. $J$ Neurophysiol 97:283-295.

Rahman M, Muhammad S, Khan MA, Chen H, Ridder DA, Müller-Fielitz H, Pokorná B, Vollbrandt T, Stölting I, Nadrowitz R, et al. (2014) The $\beta$-hydroxybutyrate receptor $\mathrm{HCA}_{2}$ activates a neuroprotective subset of macrophages. Nat Commun 5: 3944.

Rundfeldt C (1999) Characterization of the $\mathrm{K}^{+}$channel opening effect of the anticonvulsant retigabine in PC12 cells. Epilepsy Res 35:99-107.

Santhakumar V, Hanchar HJ, Wallner M, Olsen RW, and Otis TS (2006) Contributions of the $\mathrm{GABA}_{\mathrm{A}}$ receptor $\alpha 6$ subunit to phasic and tonic inhibition revealed by a naturally occurring polymorphism in the $\alpha 6$ gene. $J$ Neurosci 26:3357-3364.

Schenzer A, Friedrich T, Pusch M, Saftig P, Jentsch TJ, Grötzinger J, and Schwake $\mathrm{M}$ (2005) Molecular determinants of $\mathrm{KCNQ}\left(\mathrm{K}_{\mathrm{v}} 7\right) \mathrm{K}^{+}$channel sensitivity to the anticonvulsant retigabine. J Neurosci 25:5051-5060.

Schroeder BC, Kubisch C, Stein V, and Jentsch TJ (1998) Moderate loss of function of cyclic-AMP-modulated KCNQ2/KCNQ3 $\mathrm{K}^{+}$channels causes epilepsy. Nature 396: 687-690.

Scimemi A (2014) Plasticity of GABA transporters: an unconventional route to shape inhibitory synaptic transmission. Front Cell Neurosci 8:128.

Shorvon SD (1996) The epidemiology and treatment of chronic and refractory epilepsy. Epilepsia 37 (Suppl 2):S1-S3.

Singh NA, Charlier C, Stauffer D, DuPont BR, Leach RJ, Melis R, Ronen GM, Bjerre I, Quattlebaum T, Murphy JV, et al. (1998) A novel potassium channel gene, KCNQ2, is mutated in an inherited epilepsy of newborns. Nat Genet 18:25-29.

Singh NA, Otto JF, Dahle EJ, Pappas C, Leslie JD, Vilaythong A, Noebels JL, White HS, Wilcox KS, and Leppert MF (2008) Mouse models of human KCNQ2 and KCNQ3 mutations for benign familial neonatal convulsions show seizures and neuronal plasticity without synaptic reorganization. $J$ Physiol 586: 3405-3423.

Singh NA, Westenskow P, Charlier C, Pappas C, Leslie J, Dillon J, Anderson VE, Sanguinetti MC, and Leppert MF; BFNC Physician Consortium (2003) KCNQ2 and KCNQ3 potassium channel genes in benign familial neonatal convulsions: expansion of the functional and mutation spectrum. Brain 126: 2726-2737.

Soh H, Pant R, LoTurco JJ, and Tzingounis AV (2014) Conditional deletions of epilepsy-associated KCNQ2 and KCNQ3 channels from cerebral cortex cause differential effects on neuronal excitability. J Neurosci 34:5311-5321.

Sun J and MacKinnon R (2017) Cryo-EM structure of a KCNQ1/CaM complex reveals insights into congenital long QT syndrome. Cell 169:1042-1050.e9.

Tallian KB, Nahata MC, and Tsao CY (1998) Role of the ketogenic diet in children with intractable seizures. Ann Pharmacother 32:349-361.

Taub KS, Kessler SK, and Bergqvist AG (2014) Risk of seizure recurrence after achieving initial seizure freedom on the ketogenic diet. Epilepsia 55:579-583.

Valente-Silva P, Lemos C, Köfalvi A, Cunha RA, and Jones JG (2015) Ketone bodies effectively compete with glucose for neuronal acetyl-CoA generation in rat hippocampal slices. NMR Biomed 28:1111-1116.

van Gunsteren WF (1996) Biomolecular Simulation: The GROMOS96 Manual and User Guide, Verlag der Fachvereine Hochschulverlag AG an der ETH Zurich, Zurich, Switzerland.

Wang HS, Pan Z, Shi W, Brown BS, Wymore RS, Cohen IS, Dixon JE, and McKinnon D (1998) KCNQ2 and KCNQ3 potassium channel subunits: molecular correlates of the M-channel. Science 282:1890-1893.

Wang YJ, Fu GS, Chen FM, and Wang H (2009) [The effect of valsartan and fluvastatin on the connective tissue growth factor expression in experimental diabetic cardiomyopathy]. Zhonghua Nei Ke Za Zhi 48:660-665

Watanabe H, Nagata E, Kosakai A, Nakamura M, Yokoyama M, Tanaka K, and Sasai H (2000) Disruption of the epilepsy KCNQ2 gene results in neural hyperexcitability. J Neurochem 75:28-33. 
Wickenden AD, Yu W, Zou A, Jegla T, and Wagoner PK (2000) Retigabine, a novel anti-convulsant, enhances activation of $\mathrm{KCNQ} 2 / \mathrm{Q} 3$ potassium channels. $\mathrm{Mol}$ Pharmacol 58:591-600.

Yang L, Zhao J, Milutinovic PS, Brosnan RJ, Eger EI II, and Sonner JM (2007) Anesthetic properties of the ketone bodies $\beta$-hydroxybutyric acid and acetone. Anesth Analg 105:673-679.

Zaika O, Hernandez CC, Bal M, Tolstykh GP, and Shapiro MS (2008) Determinants within the turret and pore-loop domains of KCNQ3 $\mathrm{K}^{+}$channels governing functional activity. Biophys J 95:5121-5137.
Zhang Y, Zhang S, Marin-Valencia I, and Puchowicz MA (2015) Decreased carbon shunting from glucose toward oxidative metabolism in diet-induced ketotic rat brain. J Neurochem 132:301-312.

Address correspondence to: Dr. Geoffrey W. Abbott, Irvine Hall 232, Department of Physiology and Biophysics, School of Medicine, University of California, Irvine, CA 92697-4625. E-mail: abbottg@uci.edu 